\title{
El enfoque de género en la cooperación española para el desarrollo en el marco de la Agenda 2030*
}

\author{
The gender approach in spanish \\ cooperation for development within the \\ framework of the 2030 Agenda
}

\author{
Diana M. Verdiales López**
}

\begin{abstract}
RESUMEN
La incorporación del enfoque de género en las estrategias de la cooperación española para el desarrollo ha sido una prioridad desde sus inicios, a comienzos de la década de los noventa, tras su adhesión a la Declaración y la Plataforma para la Acción de Beijing. Las acciones emprendidas, tanto en materia legislativa como institucional, han fortalecido las acciones del gobierno de España en materia de igualdad de género, tanto en su política nacional como en su política exterior. Sin embargo, no se han producido los resultados esperados en torno a la incorporación del enfoque de género en las estrategias llevadas a cabo desde la cooperación española para el desarrollo. El presente trabajo de investigación pretende analizar el proceso de incorporación del enfoque de género en la cooperación española, destacando los avances y desafíos de dicha incorporación en las estrategias de cooperación y, sobre todo, resaltar la importancia que tiene la incorporación del enfoque de género en los planes y programas de cooperación internacional para el desarrollo, en el marco de
\end{abstract}

\footnotetext{
Este trabajo se ha realizado en el marco del Grupo de Investigación de Alto Rendimiento en Libertad, Seguridad y Ciudadanía en el Orden Internacional (INTERCIVITAS), de la Universidad Rey Juan Carlos, del cual la autora es miembro.

** Doctora en Derechos Humanos, Paz y Desarrollo Sostenible por la Universidad de Valencia, España. Responsable de Proyectos de Investigación y Cooperación del Centro de Estudios de Iberoamérica de la Universidad Rey Juan Carlos.Email: diana.verdiales@urjc.es. ORCID: https:// orcid.org/0000-0003-3275-2058. Recibido: 24 de abril de 2020. Modificado: 26 de octubre de 2020. Aceptado: 5 de noviembre de 2020.
} 
la Agenda 2030.

Palabras Clave: Enfoque de Género - Objetivos de Desarrollo Sostenible - Cooperación Internacional al Desarrollo Española.

\section{ABSTRACT}

The incorporation of the gender perspective in the strategies of Spanish cooperation for development has been a priority since its inception in the early 1990s. The actions undertaken, both in legislative and institutional matters, have strengthened the actions of the Government of Spain in matters of gender equality both in its national policy and in its foreign policy. However, the expected results have not been produced regarding the incorporation of the gender approach in the strategies carried out by the Spanish cooperation for development. Therefore, this research work aims to analyze the process of incorporating the gender approach in Spanish cooperation, highlighting the progress and challenges of such incorporation in cooperation strategies, and above all, highlighting the importance of incorporating the approach of gender in international cooperation plans and programs for development within the framework of the 2030 Agenda.

Keywords: Gender Approach - Sustainable Development Goals - International Cooperation for Spanish Development. 


\section{INTRODUCCIÓN}

El presente trabajo de investigación tiene como objetivo analizar de forma cualitativa los procesos de la incorporación del enfoque de género en la cooperación española para el desarrollo, resaltando la importancia que tiene este enfoque en la cooperación internacional. Asimismo, se analizarán las estrategias de la cooperación española para el desarrollo sostenible con enfoque de género en el marco de la Agenda 2030, resaltando los avances y desafíos de la incorporación de dicho enfoque en cooperación española al desarrollo. Por tanto, se iniciará analizando las diversas etapas por las cuales se fueron introduciendo los conceptos de igualdad de género dentro del sistema español de cooperación internacional al desarrollo. Posteriormente, se analizará la posición de la cooperación española para el desarrollo bajo el enfoque de género en las acciones implementadas para el logro de los Objetivos de Desarrollo Sostenible (oDs). Finalmente, se expondrán algunos de los proyectos de desarrollo con enfoque de género llevados a cabo por la cooperación española para el desarrollo en América Latina y se analizarán los avances y desafíos que tiene la cooperación española para el desarrollo para continuar implementando acciones de promoción de la igualdad de género en sus proyectos de desarrollo en el marco de la Agenda 2030.

Dicho análisis nos permitirá dar respuesta a algunas de las interrogantes que surgen del estudio de las acciones de la cooperación internacional española al desarrollo en materia de igualdad de género, siendo estas las siguientes: ¿Por qué es importante incorporar el enfoque de género en los programas y/o acciones de la cooperación al desarrollo? ¿Cómo se ha incorporado el enfoque de género en la Cooperación española para el desarrollo? Y, finalmente, ¿cuáles son los avances y los desafíos que se presentan para la cooperación española al desarrollo en la incorporación y promoción del enfoque de género en las acciones y estrategias necesarias para dar cumplimiento a los ods?

Para ello empezaremos por diferenciar entre el concepto de desarrollo y cooperación al desarrollo, tomando en consideración la definición de José Ángel Sotillo, el cual señala que la cooperación al desarrollo es "una herramienta, un instrumento, en este caso, diseñado para contribuir al desarrollo, mientras que el desarrollo es la finalidad, el objetivo que se pretende conseguir mediante la cooperación" (Sotillo, 2006).

Otros conceptos que podemos recoger sobre la Cooperación al Desarrollo pueden definirla como un conjunto de actuaciones internacionales para promover el desarrollo de los países menos adelantados, contribuyendo a la disminución y/o erradicación de la pobreza para promover un desarrollo humano más sostenible. Es 
importante señalar que las definiciones de la cooperación al desarrollo pueden ser variadas y múltiples, según las necesidades y/o prioridades del propio desarrollo a través del tiempo.

Es importante también tener claro el concepto de género que, como sabemos, está relacionado con las relaciones socioeconómicas y culturales entre personas de diferente sexo. Es decir, con la manera en la que nos relacionados en función de nuestro sexo, haciendo referencia a los roles, estereotipos y responsabilidades asignadas por la sociedad y lo que socialmente se espera de nosotros por el hecho de ser hombre o mujer.

Un elemento central e imprescindible en la cooperación al desarrollo es la ayuda, técnica o financiera, que destinan los países donantes para mejorar las condiciones de vida de los países en vías de desarrollo. En ese sentido, la Ayuda Oficial al Desarrollo (AOD) es definida por el Comité de Ayuda al Desarrollo de la ocDE como "la ayuda gubernamental que promueve $\mathrm{y}$ respalda al desarrollo económico y el bienestar de los países en desarrollo" (Boni, 2010). En la actualidad, podemos observar cómo los actores y estrategias en la cooperación internacional para el desarrollo han cambiado. En los últimos años, podemos ver cómo se han elevado considerablemente las donaciones privadas. Los principales actores de la cooperación internacional para el desarrollo lo conforman las empresas, las asociaciones, fundaciones e individuos, clasificándose como actores financiadores, inversionistas, gestores o intermediarios, operadores o asesores, proporcionado nuevos esquemas en la cooperación internacional para el desarrollo con estrategias de cooperación mixta.

Además de los recursos financiaros que hacen posible la puesta en marcha de los programas y acciones de la cooperación internacional al desarrollo, se considera imprescindible la incorporación de otros elementos que enriquecen y contribuyen al logro de los objetivos de desarrollo contenidos en las estrategias de la cooperación internacional. Nos referimos a una serie de acciones o enfoque relacionados con los derechos humanos, como son el enfoque basado en los derechos humanos y el enfoque de género.

El Enfoque de Derechos Humanos (rights-based approach), Naciones Unidas define como "un marco conceptual para el proceso de desarrollo humano que desde el punto de vista normativo está basado en las normas internacionales de derechos humanos y desde el punto de vista operacional esté orientado a la promoción y la protección de los derechos humanos, cuyo propósito es analizar las desigualdades que se encuentran en el centro de los problemas de desarrollo y corregir las prácticas discriminatorias, así como el reparto injusto de poder que obstaculiza el progreso en materia de desarrollo" (ACNUDH, 2006).

Por su parte, el enfoque de género es definido como un enfoque que "responde a una manera de analizar la 
realidad social, centrando la mirada en los diferentes factores que alimentan y reproducen la desigualdad existente entre mujeres y hombres en cada uno de los países socios de nuestra cooperación para poder transformarlos y cambiarlos" (AECID, 2015).

Los intentos por incluir las cuestiones de género en los proyectos de cooperación al desarrollo han ido evolucionando a lo largo del tiempo. Entre las principales estrategias o enfoques que se han desarrollado, podemos destacar las siguientes:

"Estrategia de Bienestar (años 50): La primera en incorporar a la mujer en las estrategias de cooperación, aunque basaba en su papel como madres y esposas y como beneficiarias pacíficas e indirecta de los proyectos de desarrollo (AECID, 2015).

* Estrategia de Equidad (años 70): Desarrollada tras la primera Conferencia de las Naciones Unidas para la Mujer celebrada en 1975 en México, demandando la igualdad entre los hombres y mujeres y posicionando la igualdad entre géneros en la agenda política internacional (Espinoza, 2007).

"Enfoque Mujeres en Desarrollo, MED (años 70): la cual pone en evidencia los resultados desiguales generados por el modelo de desarrollo (Espinoza, 2007 y 2014; y Molina, 2013).

* Enfoque de Género en el Desarrollo, GED (años 80): el cual analiza e intenta transformar aquellas relaciones de poder desiguales que obstaculizan el disfrute equitativo y pleno de las mujeres en todos los ámbitos del desarrollo (Espinoza, 2007 y 2013; Molina, 2013 y Pedrajas, 2014).

* Enfoque Integrado de Género/ Transversalización de Género (Gender Mainstreaming) desarrollado a finales de 1990 y principios de 2000: pretende identificar $y$ tener en cuenta las necesidades y principales problemáticas que afectan tanto a hombres como mujeres. La incorporación transversal de dicho enfoque (mainstreaming) pretende integrar la perspectiva de género tanto en el ámbito político como técnico, así como realizar los cambios funcionales y estructurales necesarios para mejorar el desarrollo y evaluación de los procesos de toma de decisiones (Fundación Mujeres, 2003).

Es importante enfatizar que, aunque ambos enfoques pudieran resultar muy parecidos en sus objetivos y estrategias, no son iguales. El enfoque basado en los derechos humanos representa un enfoque más amplio en su campo de actuación que puede incorporar dentro de sus acciones al enfoque de género. El enfoque de género refuerza la implementación y promoción de acciones relacionadas con la participación activa de la mujer en los programas o proyectos sociales elaborados bajo el enfoque de derechos humanos. Por lo que ambos enfoques pueden ser complementarios.

Pese a que España fue un país receptor de ayuda hasta principios de los años ochenta, su compromiso por apoyar a los países que más lo necesitan le llevó a emprender una serie de acciones institucionales y políticas para 
fortalecer su política de cooperación internacional al desarrollo. A partir de los años ochenta hemos visto un papel muy activo de la cooperación internacional española en sus estrategias de gestión interna, mejorando y ampliando progresivamente su estructura organizacional e incorporando enfoques de trabajo como el sectorial y el transversal. Ambos enfoques claves para la introducción, defensa y promoción de la igualdad de género.

Después de solicitar, en 1983, su salida de la lista de países receptores de Ayuda Oficial al Desarrollo (AOD) del Comité de Ayuda al Desarrollo (CAD), España inicia sus primeras acciones en la cooperación internacional, creando en 1985 la Secretaria de Estado para la Cooperación Internacional y para Iberoamérica (SECIPI) como el primer órgano político especializado en cooperación al desarrollo, responsable de la dirección, programación, control y evaluación de la política española de cooperación al desarrollo (Gómez, 2010).

Con el propósito de mejorar sus acciones con el exterior, el gobierno de España inició una serie de acciones encaminadas a mejorar la calidad, el rigor, la transparencia y la eficacia de la cooperación española. Para ello creó, en 1988, la Agencia Española de Cooperación Internacional (AECI) adscrita a la SECIPI. Y ese mismo año se creó la Oficina de Planificación y Evaluación (OPE), que permitiría el desarrollo de un sistema de evaluación mediante el fortalecimiento de los recursos humanos.

Desde la creación de la Ley de Cooperación Internacional para el Desarrollo, bajo el Real Decreto 23/1998, se han ido introduciendo las cuestiones relativas a la igualdad de género en la legislación española (воE, 1998a). Dicha Ley tiene, entre sus principios fundamentales, la participación de la ciudadanía en condiciones de igualdad tanto para hombres como para mujeres, sin discriminación alguna por motivos de sexo, raza, cultura o religión.

Como parte de los compromisos del gobierno español de continuar apoyando a los países menos adelantados y para mejorar los proyectos de cooperación con enfoque de género, realizó modificaciones en su principal organismo de cooperación internacional: la Agencia Española de Cooperación. Así, en 2007, la Agencia Española de Cooperación Internacional pasó a ser la Agencia Española de Cooperación Internacional para el Desarrollo (AECID), con el objetivo de adaptarse mejor a las mejoras de los servicios públicos establecidos en la ley 28/2006 de las agencias estatales (Tomás, 2013), creando, dentro de esta nueva Agencia, el Departamento Sectorial y de Género (Jiménez, 2014). Asimismo, se elaboró la Metodología de Evaluación de la Cooperación Española en 1998, la cual formaría parte del plan de evaluaciones de la cooperación al desarrollo, como parte de las mejoras organizativas de la cooperación al desarrollo (Espinoza, 2007 y Arguilés, 2014). 
Es importante señalar que previo a la promulgación de la Ley de Cooperación Internacional al Desarrollo, la planificación de la Política de Cooperación al Desarrollo española se llevaba a cabo mediante la elaboración de Planes Anuales de Cooperación Internacional (PACI). Una vez aprobada dicha Ley, la planificación pasó a organizarse mediante un Plan Director de carácter cuatrienal, el cual fija las directrices generales de actuación, y un Plan Anual que marca los objetivos, recursos y prioridades a desarrollarse anualmente en el ámbito de la cooperación al desarrollo.

Durante los primeros años del 2000, el gobierno español realizó un importante esfuerzo de expansión y modernización de sus planes e instrumentos en materia de Cooperación Internacional al Desarrollo. Creando, para ello, directrices nacionales bajo estrategias sectoriales regidas por el I Plan Director de la Cooperación para el Desarrollo en 2001. Estas acciones de modernización, aunadas al crecimiento económico sostenido, hicieron posible una mayor participación de España en el ámbito de la Cooperación Internacional al Desarrollo, desempeñando un papel clave en la cooperación multilateral.

Continuando con las estrategias de modernización, en 2005 se creó la Dirección General de Planificación y Evaluación de Políticas para el Desarrollo (DGPOLDE), como la unidad especializada para institucionalizar la perspectiva de género e impulsar el "análisis de género" en los proyectos de cooperación (Espinoza, 2007). Estas estrategias de modernización de la Cooperación Internacional al Desarrollo española permitieron cambiar los enfoques en la actuación de sus programas, pasando de enfocarse en las situaciones (pobreza, desigualdad, etc.) a enfocarse en las personas (mujeres, niños, indígenas, migrantes, etc.); de considerar como logros el mero cumplimiento de los objetivos y el desembolso del presupuesto a generar un empoderamiento en la población beneficiada; de fomentar un método de aprendizaje basado en lo didáctico y lo instructivo, para pasar a un método de aprendizaje experimental (Boni, 2010).

Pese a los esfuerzos de modernización, la obsolescencia de los sistemas administrativos y de coordinación entre las distintas dependencias que conforman la cooperación al desarrollo española, dificultaron el avance en la implementación de enfoques transversales efectivos y eficientes como el enfoque de género. Todo ello aunado a los efectos producidos por la crisis económica del 2008, imposibilitaron la transversalización del enfoque de género en las acciones y programas de la cooperación española al desarrollo (Jiménez, 2015).

Empero, consideramos de gran importancia que se continúe avanzando en la incorporación del enfoque de género en las acciones y programas de 
la cooperación española al desarrollo, en consonancia con lo establecido en los Objetivos de Desarrollo Sostenible. Por ello, en las siguientes líneas se abordará la importancia de la incorporación del enfoque de género en la cooperación española al desarrollo.

\section{II.- LA IMPORTANCIA DE LA INCORPORACIÓN DEL ENFOQUE DE géNero en la CoOperación InTERnaCional al Desarrollo ESPAÑOLA}

Las cuestiones relativas a la igualdad de género han sido siempre un tema prioritario para la política nacional española. Pero cobran mayor importancia a partir de los años noventa, tanto en la agenda nacional como en la agenda internacional (Espinoza, 2007 y 2014). Prueba de ello es su adhesión a ocho tratados internacionales fundamentales y a 10 protocolos facultativos sobre igualdad de género y no discriminación de la mujer (MAEC, 2020).

En el ámbito internacional, España ha suscrito el compromiso por la igualdad entre mujeres y hombres tanto en la Convención sobre la Eliminación de todas las Formas de Discriminación contra la Mujer (CEDAw, 1979), como en la Declaración y la Plataforma para la Acción de Beijing (1995), en los Objetivos de Desarrollo del Milenio (2000) y, posteriormente, en los Objetivos de Desarrollo Sostenible (2015) (Tomás, 2013). $\mathrm{Y}$ a nivel europeo, según lo suscrito en la Carta de los Derechos Fundamentales, como en el Tratado de Funcionamiento recogido en el Tratado de la UE, en el Tratado de Roma (1957) y específicamente en el Convenio del Consejo de Europa sobre Prevención y Lucha contra la Violencia contra las Mujeres y la Violencia domestica de 2011 (Convenio de Estambul). Asimismo, forma parte del Compromiso Estratégico para la igualdad entre mujeres y hombres 2016-2020 en consonancia con el Pacto Europeo para la Igualdad de Género 2011-2020 (Espinoza, 2014).

Asimismo, podemos encontrar cómo en su legislación nacional se recogen las cuestiones relativas a la igualdad de género. Tal como se señala en el Artículo 1 de la Constitución Española, el cual reconoce la igualdad como uno de los valores superiores del ordenamiento jurídico; en el Artículo 9.2 se hace mención sobre las condiciones para promover la libertad y la igualdad entre los individuos, y en el Artículo 14 de la Carta Magna se

1 Establece en su artículo 119 el principio de igualdad en la retribución salarial entre hombres y mujeres que realizan el mismo trabajo, prohibiendo la discriminación salarial por razón de sexo. AECID (2015), “Guía de la AECID sobre la Transversalización del Enfoque de Género", Madrid, España, p. 25. 
prohíbe la discriminación por razones de sexo.

En materia de Cooperación Internacional al Desarrollo, la participación activa de España en las conferencias internacionales como la I Conferencia Mundial de las Mujeres de la onu, llevada a cabo en México en 1975; II Conferencia de la Mujer celebrada en Copenhague en 1980 y fundamentalmente la III Conferencia de la Mujer celebrada en Nairobi (1985), le ha llevado a considerar los temas relativos a la igualdad y equidad de género, y vincularlos estratégicamente en su política de cooperación al desarrollo (MAEC, 2007).

De manera más oficial, el enfoque de género se hace presente por primera vez en el Plan Director 2005-2008 y en la Ley 23/1998 de Cooperación Internacional para el Desarrollo. De estos dos documentos se deriva la Estrategia de "Género en Desarrollo", la cual se constituye como un instrumento fundamental para promover la igualdad efectiva entre hombres y mujeres y sobre todo para poner en práctica no solo los lineamientos expuestos en el Plan Director 2005-2008 y en la Ley 23/1998, sino también en lo contenido en la Ley 3/2007 para la igualdad efectiva de mujeres y hombres (Tomás, 2013 y Espinoza, 2014). Dichas directrices se ponen de manifiesto en las primeras

2 Para mayor información ver en: https:// www.aecid.es/Centro-Documentacion/ Documentos/Planificación $\% 20$ estratégica $\% 20$ por $\% 20$ sectores/ EstrategiaGENEROdes.pdf experiencias piloto que se llevaron a cabo bajo las Oficinas Técnicas de la cooperación española conjuntamente con organizaciones lideradas por mujeres en países como Guatemala, Nicaragua, República Dominicana, entre otros, incluyendo en ellos la transversalización del enfoque de género.

Leyes como la Ley contra la violencia de género aprobada del 2004, la Ley de Acción y Servicio Exterior de 2014 y el Pacto de Estado 2018-2022, contienen medidas específicas que se integran en la política exterior española en la lucha contra la trata y la violencia. Por su parte, la Ley de Acción y Servicio Exterior de 2014, considera a la igualdad de género como uno de los ámbitos más importantes en las acciones con el exterior. Esta ley tiene entre sus principios "la promoción de la igualdad entre hombres y mujeres, y a la erradicación de la violencia de género, destacando la protección de las mujeres españolas víctimas de violencia de género y de sus hijos e hijas en el exterior" (MAEC, 2020).

Estas acciones han producido importantes avances dentro de la cooperación española para el desarrollo en la promoción de las cuestiones de igualdad de género. A nivel internacional podemos ver cómo España se encuentra por encima de la media europea en las acciones de igualdad de género. Según el Índice Europeo de la Igualdad de Género ${ }^{3}$ de 2019 sitúa a España

3 https://eige.europa.eu/gender-equalityindex/2019 
como noveno Estado Miembro de la Unión Europea con los mejores resultados. Por su parte, el Foro Económico Mundial, en su Global Gender Gap Report 2020, situó a España en el número ocho a nivel global. Asimismo, el Índice de Género de los Objetivos del Desarrollo Sostenible (sDG Gender Index/Equal Measures 2030), sitúa a España en el puesto 23 de 129 países examinados (MAEC, 2020).

Como ya hemos hecho mención, en 2007 se publicó la Estrategia de Género en Desarrollo para consolidar las acciones de género contenidas en el II Plan Director de la Cooperación y reafirmarlas en el III Plan Director (Tomás, 2013); así como el Plan de Actuación Sectorial de Género. Estos documentos, junto a los sucesivos Planes Directores, constituyen un marco de referencia en materia de igualdad de género en la cooperación española para el desarrollo (Espinoza, 2014).

El Plan de Actuación Sectorial de Género y Desarrollo señala cinco líneas de trabajo estratégicas y prioritarias para la cooperación, como son: la participación social y política; la lucha contra la violencia de género; los derechos sexuales y reproductivos; la mujer y construcción de paz, y los derechos económicos y laborales. Asimismo, se incorporaron a dicho Plan dos líneas de fortalecimiento institucional, como son la transversalización y la institucionalización del enfoque de género (AECID, 2015).

En ese sentido, se pusieron en marcha programas a nivel de postgrado en materia de género para promover los derechos de las mujeres tanto en el tercer sector, como del personal de gobierno de las dependencias adscritas a la cooperación internacional, y para el público en general. Con las nuevas estrategias de fortalecimiento de las cuestiones de género señalados en el Plan de Actuación se requería que los gestores de proyectos tomaran en consideración las diferencias y desigualdades existentes entre hombres y mujeres en los países socios donde se cooperaba. Esta visión requirió la participación de los hombres en los proyectos de cooperación, para trabajar con ellos una de las causas estructurales de la desigualdad de género, abordando temas como los roles en la familia o la percepción tradicional de la mujer en la sociedad. En este sentido, la Cooperación Española al Desarrollo hace énfasis en que la igualdad entre hombres y mujeres es la base para la generación de desarrollo.

En esta línea, la Cooperación al Desarrollo Española elaboró una serie de actuaciones para hacer más efectivo el proceso de transversalización de las cuestiones de género en los programas desarrollados con los países socios. Trabajando básicamente desde lo formal hasta lo real, es decir incorporando la igualdad formal mediante la adecuación legislativa nacional con la normativa internacional y su vinculación con los derechos económicos, sociales y culturales, así como los sexuales y reproductivos, y la igualdad real con la promoción de las políticas de género 
y acciones concretas y específicas sobre el combate de todas las formas de violencia y de discriminación contra las mujeres. Requiriendo para ello el desarrollo de tres pasos fundamentales (Tomás, 2013):

1. Que las cuestiones de género pasen a ser prioridad política en la agenda principal y así se refleje en sus documentos y declaraciones del país socio.

2. Que se acompañe con un proceso real de institucionalización a medio plazo.

3. Que haya una efectiva transversalización desde la planificación hasta la gestión,el seguimiento y la evaluación de los programas implementados desde la cooperación internacional al desarrollo.

Es importante enfatizar que los Planes Directores de la Cooperación para el Desarrollo Española (20012004,2005-2008, 2009-2012, 2013-2016 y 2018-2021), fungen como el instrumento más importante de planificación que guía las intervenciones de la cooperación española para el desarrollo en el exterior. En ella se recogen las estrategias de igualdad de género que serán promovidas en los programas de cooperación, siendo a partir del II Plan cuando se incorporan el enfoque de Género en el Desarrollo (GED) y la transversalización de género. Los cuales detallaremos a continuación:

\section{Plan Director de la Cooperación para el Desarrollo Española 2001- 2004:}

En este Plan Director, la igualdad entre hombres y mujeres es una de las prioridades horizontales. Ello demanda el establecimiento de los vínculos entre la igualdad de género y las demás estrategias de la Cooperación para el Desarrollo española. Exhortando la inclusión de los aspectos relativos al enfoque de género en la formulación, programación, ejecución y evaluación de todos los programas y proyectos, tanto sectoriales como geográficos desarrollados en el marco de la cooperación española al desarrollo.

La integración de la perspectiva de género en este plan repercute en las actuaciones de la Cooperación Española al Desarrollo, a través de la realización de análisis e investigaciones sobre los efectos de las políticas de desarrollo en las mujeres, la incorporación de indicadores de impacto y la obtención de datos desagregados por sexo, así como en la formulación y ejecución de proyectos específicos para la eliminación de la discriminación, la integración y la participación de la mujer en los procesos de desarrollo (Tomás, 2013).

\section{Plan Director de la Cooperación para el Desarrollo Española 2005- 2008:}

A partir de este Plan Director, la Cooperación al Desarrollo Española asume el enfoque de Género en Desarrollo (GED) con una doble prioridad, tanto horizontal como especifica (Tomás, 2013). Este enfoque se contrapone y busca superar algunas limitaciones del Enfoque de Mujeres en el Desarrollo (MED), vigentes en las políticas 
de desarrollo desde los años ' 70 , en los que se perpetuaban estereotipos y roles tradicionales de mujeres y hombres, y no se atendían los intereses estratégicos de género de las mujeres. El enfoque Género en Desarrollo busca superar los problemas derivados de relaciones desiguales de poder entre las personas que perpetúan la pobreza, las desigualdades y el crecimiento económico insostenible en el mundo (AECID, 2019).

\section{Plan Director de la Cooperación para el Desarrollo Española 2009- 2012:}

Este plan continúa dando prioridad a las cuestiones de igualdad de género tanto como una prioridad horizontal y sectorial, para dar seguimiento y continuidad a las acciones emprendidas en el plan anterior. A diferencia de este, el III Plan intenta aplicar el enfoque de Derechos Humanos de las Mujeres y vincular el enfoque de género en desarrollo a los contenidos de la Eficacia de la Ayuda, teniendo como principal objetivo a nivel sectorial, alcanzar el pleno ejercicio de los derechos humanos y el empoderamiento de las mujeres mediante la construcción de una ciudanía con perspectiva de género (Jiménez, 2014).
IV Plan Director de la Cooperación para el Desarrollo Española 20132016:

En este Plan Director se realizan tres referencias claves en relación con la igualdad de género y los derechos de las mujeres en la consolidación de procesos democráticos y de Estado de Derecho (Espinoza, 2014):

1. Impulsar políticas públicas que promuevan la igualdad de género entre hombres y mujeres.

2. Trabajar para que el sistema de justicia sea accesible en igualdad de condiciones para todos los ciudadanos y garantice la tutela judicial efectiva y el derecho a la defensa independiente, de calidad y gratuita, con especial atención a mujeres y niñas, y colectivos en riesgo de discriminación y vulnerabilidad, como los indígenas y los afrodescendientes.

3. Promover los Derechos de las Mujeres y la Igualdad de Género, de manera formal y real y aplicar acciones específicas para paliar todas las formas de violencia y discriminación contra las mujeres y las niñas, con especial atención a situaciones como la trata y explotación sexual o la mutilación genital femenina y sus consecuencias; la discriminación y vulnerabilidad de las mujeres en la economía y el trabajo, sobre todo en lo relativo a la economía de los cuidados, las trabajadoras del hogar o en la economía informal y las mujeres trabajadores en las áreas rurales. 


\section{$V$ Plan Director de la Cooperación para el Desarrollo Española 2018 2021:}

Este Plan centra sus acciones en el cumplimiento a la Agenda 2030 en lo relativo a la promoción de la igualdad de género y el empoderamiento de las mujeres y las niñas, así como en el apoyo y protección para que puedan ejercer plenamente sus derechos. Al igual que en los Planes Directores anteriores, en este plan se sigue promoviendo el Enfoque de Género en el Desarrollo, así como la transversalización de género en las intervenciones de desarrollo, para continuar avanzando para generar cambios en la cultura institucional y en todas aquellas acciones para eliminar las desigualdades de género y combatir la violencia de género en todas sus formas, en los diversos ámbitos, tanto social, como político, económico y laboral(MAEC, 2020).

Según la Agencia Española de Cooperación Internacional para el Desarrollo, existen numerosas razones que avalan la importancia de la incorporación del enfoque de género en la cooperación internacional al desarrollo, entre las que señalaremos las siguientes (AECID, 2015):

" La igualdad entre mujeres y hombres es la base del desarrollo.

* Existe una situación de desigualdad social entre mujeres y hombres con respecto a las actividades que desempeñan, a su acceso a recursos y el control de los mismos, y a su participación en la toma de decisiones.

"Las estrategias de desarrollo no son neutras en relación con el género al tener consecuencias positivas y negativas diferenciadas para hombres y mujeres.

* Las acciones de desarrollo son más eficaces si tienen en cuenta las diferencias y desigualdades entre mujeres $y$ hombres.

* La invisibilización de las mujeres en las intervenciones de desarrollo complica la definición de las estrategias para apoyar la satisfacción de sus necesidades prácticas e intereses estratégicos.

*Analizar los contextos y la realidad desde el género implica no considerar a todas las mujeres y hombres como iguales. Las relaciones de género interactúan simultáneamente con otras características o condiciones que pueden duplicar o triplicar las desigualdades y discriminaciones como el origen étnico o nacional de las personas, el grupo social, la edad o la orientación sexual, entre otras.

* Las mujeres no son un grupo vulnerable (representan más de la mitad de la población mundial), pero debido a las múltiples discriminaciones de las que son objeto están en condiciones de exclusión y que a veces enfrentan mayor vulnerabilidad.

* Trabajar con mujeres no significa que automáticamente se tienen en cuenta los aspectos relacionados con la desigualdad de género desde un enfoque de derechos humanos, 
pudiéndose impulsar proyectos exclusivos para mujeres que tengan un efecto muy negativo en las relaciones de género y refuercen su posición de subordinación.

"Los hombres son un elemento clave para el cambio en las relaciones de género. Es imprescindible trabajar con ellos para eliminar las causas estructurales de la desigualdad de género y modificar la percepción "tradicional" que tanto hombres como mujeres tienen de su "rol" de género.

* Los esfuerzos para corregir las desigualdades a través de proyectos dirigidos a mujeres pueden resultar contraproducentes si los hombres no son informados e incluidos a lo largo del proceso. (AECID, 2015)

Tal como indica el documento marco de VENRO sobre la incorporación del enfoque de género en la cooperación al desarrollo, hay coincidencias en los debates de género en que las mujeres están estructuralmente discriminadas en todas las sociedades y sujetas a cargas extraordinarias; en que las mujeres juegan un rol clave en los procesos de desarrollo, por ser las que aseguran la sobrevivencia familiar, siendo por tal portadoras claves de la promoción del desarrollo económico y social, y en que para lograr la equidad de género se requiere de la implementación de estrategias complementarias, como la promoción de la mujer y el enfoque transversal de género (venro, 2009), por lo que creemos que se debe continuar trabajando en la incorporación de enfoques de género en los planes y programas tanto nacionales como internacionales, a través de la Cooperación Internacional al Desarrollo.

\section{III.- La Cooperación Española al Desarrollo con enfoque de género en el marco de la Agenda 2030}

Con la llegada, en 2015, de la nueva Agenda para el Desarrollo Sostenible, las estrategias de igualdad de género se vieron reforzadas, cobrando mayor importancia al estar contenidas de forma más concreta en 2 de los 17 Objetivos de Desarrollo Sostenible, como son el Objetivo 5 sobre la igualdad de género y el Objetivos 10 sobre la reducción de las desigualdades. Aunque son estos los Objetivos que abordan de manera directa las cuestiones de género en el desarrollo, las relativas a la mujer están contenidas de forma indirecta en prácticamente todos los Objetivos de Desarrollo Sostenible.

Como hemos visto en el apartado anterior, la Cooperación Española al Desarrollo ha impulsado las políticas de igualdad de género tanto dentro como fuera de sus fronteras, en cumplimiento con la Plataforma de Acción de Beijing y la Agenda 2030. Estas acciones han sido posibles por la implementación de Estrategia de Género en Desarrollo, que ha permitido incorporar el enfoque de género en la planificación, gestión y evaluación, y por las acciones específicas implementadas para garantizar el pleno ejercicio de los derechos de las mujeres mediante 
el enfoque transversal de género en sus políticas sectoriales (MAEC, 2020).

Entre las acciones internas que se han desarrollado en la Cooperación Española al Desarrollo, destaca la introducción en su V Plan Director (2018-2021) de las acciones para el cumplimento de la Agenda 2030. Específicamente se han introducido acciones de igualdad de género y el empoderamiento de las mujeres y niñas, así como la promoción de sus derechos como mejor forma de avanzar hacia el ejercicio de su ciudadanía y en la lucha contra la pobreza. Instando a los países con los que colabora a impulsar programas y acciones que incluyan el enfoque transversal de género, en consonancia a lo establecido en su IV y V Plan Director, así como en el Plan Estratégico y en la Guía de Transversalización del Enfoque de Género, promovidos por la Agencia Española de Cooperación Internacional para el Desarrollo

Otras acciones que han promovido la política de igualdad de género de la Cooperación Española al Desarrollo en el exterior han sido la participación activa de España en la Agenda de Mujeres, Paz y Seguridad y el nombramiento, en 2018, de una Embajadora en Misión Especial para el fomento de políticas de igualdad de género y conciliación, para dar un paso más en la aplicación del enfoque de género en la política exterior española.

En la Agenda de Mujeres, Paz y Seguridad, de la cual España ha sido impulsora, se actúa con el firme convencimiento de que para construir sociedades pacíficas y para lograr un desarrollo sostenible es clave la participación de las mujeres en todas las fases de los conflictos (MAEC, 2020).

Por todo lo anterior, España se ha convertido un referente internacional en materia de igualdad de género ${ }^{4}$.Sin embargo, los efectos de la crisis económica de 2008, han conseguido frenar los avances conseguidos por España tanto en sus políticas sociales como en sus estrategias de enfoque de género de su Cooperación Internacional. Ya que, según el último informe sobre las políticas sociales en España, los problemas derivados de los ajustes presupuestarios en las políticas internas han conseguido aumentar la desigualdad, afectando más a las mujeres de este país. En este sentido, según el último registro de desempleo, en 2017 las mujeres representaban un $58 \%$ de la tasa de paro y la diferencia salarial seguían siendo altas, teniendo como media una diferencia salarial de $€ 5.941$, equivalente a un 19.3\% en 2013 (Gobierno de España, 2018). En cuanto al tiempo de dedicación al cuidado del hogar, la diferencia también continúa

4 Así lo señala el informe realizado por el Banco Mundial en 2016, titulado "Women, Business and the law", situando a España como uno de los países con mejor marco normativo para garantizar la plena igualdad legal de oportunidades. Gobierno De España (2018), "Informe de España para el Examen Nacional Voluntario 2018". Gobierno de España, Madrid, p. 48. 
siendo considerable, ya que las mujeres destinan más del doble de tiempo a los cuidados familiares y domésticos que los hombres, siendo un grave problema para su desarrollo personal y profesional, ya que pese a su alta formación académica $(60.12 \%$ con estudios universitarios en 2016) la mayoría de estas, un $75 \%$, cuenta con trabajos a tiempo parcial y de baja remuneración (Gobierno de España, 2018).

Como ya hemos hecho mención, los problemas de desigualdad de género en España han sido un tema prioritario, pero específicamente los relativos a la violencia de género. En este sentido, el país fue pionero a nivel internacional en la aprobación de una Ley contra la Violencia de Género. $\mathrm{La}$ Ley Orgánica 1/2004 sobre las medidas de Protección Integral contra la Violencia de Género, se creó con el propósito de trabajar de forma integral y multidisciplinar en las medidas de sensibilización, prevención y detección de la violencia de género. Es necesario precisar que meses previos a la aprobación de dicha Ley, en 2003, se había puesto en marcha un programa para contabilizar las muertes por violencia de género. En un inicio solo se incluían a las mujeres asesinadas por violencia de género, siendo hasta 2013 cuando se empieza a incluir a los menores asesinados víctimas de violencia de género.

Una de las formas de prevención más importantes ha sido la denuncia. En 2017 se registraron más de 13.500 denuncias diarias por violencia de género (Gobierno de España,2018), de las cuales en 2016 el 28,28\% de las víctimas que habían presentado una denuncia previa terminaron falleciendo (OXFAM y unicef, 2015), lo que significa que este problema social continúa siendo uno de los grandes retos para el país. Por ello, se vislumbra muy lejana la consecución de la meta propuesta por España en los ods de reducir a un 10\%, en 2020, las víctimas mortales por violencia de género con denuncia previa.

En los últimos meses hemos visto con agrado la noticia sobre la recuperación del Ministerio de Igualdad y su integración en la Vicepresidencia de Gobierno, como tema prioritario para el Gobierno nacional. En este sentido, el gobierno actual pretende trabajar en el nuevo Pacto Estratégico de Igualdad de Oportunidades para incluir las metas y objetivos señalados en la Agenda 2030, específicamente lo descrito en el Objetivo 5, al tiempo en que se trabaja en la II Estrategia Nacional para la Erradicación de la Violencia Contra la Mujer (2018-2022), en consonancia con los compromisos internacionales adquiridos.

A pesar de ello, algunas organizaciones del tercer sector, como Intermon Oxfam y Unicef Comité Español, señalan la necesidad de continuar trabajando en estrategias que promuevan la igualdad de género, tales como: la puesta en marcha de políticas y medidas específicas, a través de la vigilancia y la penalización de empresas que lleven a cabo prácticas discriminatorias, incumpliendo con la legislación vigente para reducir la brecha 
salarial entre mujeres y hombres $(15 \%$ en 2020 y su completa eliminación para el 2030); fortalecer las capacidades, financieras, institucionales y técnicas tanto a nivel nacional como autonómico, para promover la igualdad de género y los derechos de las mujeres; ampliar y mejorar la existente disparidad de los servicios autonómicos de atención integral a las víctimas de violencia de género, y desarrollar un conjunto de medidas, entre las que se encuentran ampliar las relativas a la conciliación de la vida familiar y laboral, así como trabajar en la concepción de la familia, removiendo estereotipos y prejuicios sobre los roles familiares, para mejorar los roles de las mujeres y los hombres en las tareas domésticas y de cuidado familiar (Angulo, 2015).

Estos aspectos sobre la problemática de la desigualdad de género en España sin duda afectan de alguna manera sus estrategias de Cooperación Internacional al Desarrollo. Si este problema no es resuelto internamente, resulta poco probable que se pueda trabajar externamente promoviendo la igualdad de género en terceros países. Aun así, España basa sus estrategias de género en la cooperación al desarrollo mediante la utilización de los principios básicos de Naciones Unidas relativos a la igualdad y a la no discriminación. Asimismo, incorpora los principios emanados de la Declaración de París relativos a la apropiación y al compromiso adquirido con los países socios para que elaboren y refuercen sus políticas y estrategias de desarrollo; al alineamiento y sintonía con las políticas de desarrollo del país socio; a la armonización de las acciones para que se ejecuten de manera coordinada y tengan procesos y resultados transparentes y eficientes; a la mutua responsabilidad para la utilización de los recursos de la manera más eficaz y eficiente, y a la gestión orientada a los resultados para establecer mecanismo entre ambas partes para el seguimiento y la evaluación de los resultados. (MAEC, 2007).

Por otro lado, también es importante seguir trabajando en la transversalización del enfoque de género en las políticas nacionales. Ello permitirá avanzar hacia el logro de los Objetivos de Desarrollo Sostenible. En este sentido, es importante conocer que dentro de los oDs se identifica claramente la incorporación transversal de la perspectiva de género en los 17 objetivos de desarrollo. Sin embargo, de los 232 indicadores contenidos en estos, solo 54 están relacionados con las cuestiones de género. Es decir, solo 6 de los 17 oDs cuentan con indicadores claros sobre género (ODS1, ODS3, ODS4, ODS5, ODS8 y ODS16); 5 los contienen en menor medida (ODS2, ODS10, ODS11, ODS13 y ODS17), y 6 no hacen referencia alguna a las cuestiones de género (ODS6, ODS7, ODS9, ODS12, ODS14 y ODS15) (UN WOMAN, 2018).

Estamos convencidos que para que se avance hacia la consecución de los oDs con enfoque de género, es necesario el compromiso no solo del gobierno, sino del resto de los actores sociales, 
tales como la sociedad civil, el sector académico, el empresarial y el de las ONGs. Principalmente por parte del gobierno español se debe mantener la ayuda internacional al desarrollo, por lo menos la aportación del $0,7 \%$ del PNB en forma de ayuda oficial al desarrollo, de la cual el 0,15-0,20\% debería ir dirigida a los países menos adelantados (Alonso, 2015). Consideramos que el compromiso para hacer posible las metas contenidas en la Agenda 2030 tanto a nivel nacional como internacional, requiere del esfuerzo de todos los sectores que conforman un país y sobre todo de una coherencia en las políticas nacionales que integren, de forma equilibrada, las dimensiones sociales, económicas y ambientales desde la perspectiva de género.

\section{Avances y desafíos de la CoOperación Española al Desarrollo con enfoque de Género en los Objetivos de Desarrollo Sostenible}

Como hemos explicado en las líneas anteriores, la Cooperación Española para el Desarrollo ha implementado una serie de cambios estructurales para introducir el enfoque de género en sus planes y estrategias de actuación. Los cambios más importantes fueron la introducción del Enfoque de Género en Desarrollo (GED) con un carácter de doble prioridad: como prioridad horizontal y como sector específico, en el II Plan Director de la Cooperación Española para el Desarrollo (2005-2008). Estas prioridades se fueron consolidando con la elaboración de la Estrategia de Género en Desarrollo de la Cooperación Española, creada en 2007 y se reafirmaron en el III Plan Director de la Cooperación Española para el Desarrollo (2009-2012).

Estos cambios, aunados al aumento de recursos destinados al sector, la creación de un departamento específico sobre género, la elaboración del primer Plan de Actuación de Género de la AECID y la aprobación de la Ley 3/2007 para la Igualdad Efectiva de Mujeres y Hombres (LOIEHM) y del Plan Estratégico de Igualdad de Oportunidades 2008-2011, los cuales cuentan con un eje específico para la política exterior y la cooperación al desarrollo; sin duda representan el compromiso del Gobierno de España con la igualdad de género y la lucha contra la pobreza mediante el empoderamiento de la mujer.

Algunos de los programas más emblemáticos y exitosos que se han desarrollo en el marco de la Cooperación Española para el Desarrollo con enfoque de género, son los siguientes ${ }^{5}$ :

5 Mayor información: https://www.aecid.es/ ES/sectores-de-cooperación/género 
1.- Programas Emblemáticos (América Latina):

a) Programa: Prevención de la Violencia y Crimen Contra la Mujer y la Niñez

País de actuación: Guatemala. Importe: 15 millones de euros. Total proyecto: 16.600 .000 euros.

b) Programa: Fortalecer sistemas integrales de protección de niños, niñas y adolescentes ante la trata y tráfico con fines de explotación sexual comercial, contribuyendo al ejercicio de su derecho a una vida libre de violencia.

País de actuación: Bolivia. Importe: 2.500 .000 euros.

c) Programa: Más mujeres campesinas, jóvenes rurales y trabajadoras urbanas del sector maquila, transforman ideas, creencias y prácticas promoviendo su autonomía individual y colectiva para el ejercicio de sus derechos económicos y derecho a una vida libre de violencia.

País de actuación: Nicaragua.

Importe: 2.500 .000 euros.

d) Programa: Por el derecho de las mujeres, adolescentes y niñas a una vida libre de violencias, en las regiones de Cusco y Lima.

País de actuación: Perú. Importe: 2.500 .000 euros.

2.- Programas de ámbito bilateral:

a) Programa: Comprehemsive Agreement in Respect of Human Rights and International Humanitarian LaW (SULONG CARHIHL)
País de actuación: Filipinas. Importe: de $315.978 €$ en 2017.

b) Programa: Programa de Salud Sexual y Reproductiva, proyecto plurianual iniciado en 2009.

País de actuación: Mali.

Importe: 2,500,000.00€.

c) Programa: apoyo al Ministerio de la Población, Promoción de la Mujer y Protección de la Infancia, para la implementación de la Política Nacional de Género y la construcción de la Casa de la Mujer en la región de Maradí.

País de actuación: Níger.

Importe: 1,000,000.00€.

3.- Algunos programas de ámbito global:

a) Iniciativa Global de Ciudades Seguras, ejecutado con ONU Mujeres.

b) Programa conjunto de servicios esenciales para mujeres víctimas de violencia de género, ejecutado por FNUAP.

c) Programa de fortalecimiento de políticas de inclusión social, género y prevención de la violencia sexual para poblaciones en situación de vulnerabilidad, en el ámbito de la discapacidad.

d) Programa WE DECIDE, en colaboración con el Fondo de Población de NN.UU.

Pese a las acciones de promoción y difusión de la igualdad de género y empoderamiento de la mujer llevadas a cabo por la Cooperación Española al Desarrollo en colaboración con los países socios y de organismos 
internacionales, todavía existen enormes desafíos en la plena incorporación del enfoque transversal de género en los programas y acciones llevados a cabo en consonancia con la Agenda 2030.

Uno de los principales problemas detectados es la falta de formación del personal técnico de la Agencia Española de Cooperación Internacional para el Desarrollo. Asimismo, se observa una falta de liderazgo y compromiso por parte de personal del Estado, que asume la responsabilidad política de promoción de la equidad de género en los programas y proyectos (Tomás, 2013 y Espinoza, 2014), poniendo en riesgo la institucionalización de este enfoque de género en los programas de cooperación si no se formaliza y asegura su sostenibilidad con medidas operativas, y la consolidación de la restructuración de la Agencia Española para el Desarrollo.

Según el Informe de 2018 sobre el Enfoque de Género en la Política de Cooperación Española, se ha producido, en los últimos años, una significativa pérdida del personal especializado en género tanto en sede como en terreno, provocando un impacto negativo en la aplicación y evaluación de la perspectiva de género en los programas implementados (MAEC, 2018a).

Otra cuestión clave a analizar es la forma en la que se está introduciendo la estrategia de transversalización y sectorialización de la perspectiva de género en los programas de la Cooperación Española para el Desarrollo, ya que en la mayoría de los procesos implementados por la Agencia Española de Cooperación Internacional para el Desarrollo se ha optado por la transversalización más que por la realización de acciones específicas, lo que ha generado que se incorporare el enfoque de género dentro de otros sectores sin priorizar como sector propio del país socio, provocando una ausencia de incorporación real de los enfoques en la formulación, ejecución, seguimiento y evaluación de las intervenciones realizadas (Jiménez, 2015). Por lo que de los 21 países que cuentan con los citados Marcos de Asociación País (MAP), solo 6 de ellos han priorizado género como orientación estratégica (MAEC, 2018a). Todo ello redunda en la dificultad de implementar estratégicamente el enfoque transversal de género y poder dejar las bases de medición y evaluación de los objetivos en los planes de los países socios. En este sentido, el Grupo de Trabajo de Género y Desarrollo recomienda hacer una clara distinción entre $\mathrm{Mu}$ jeres en Desarrollo (MED) y Género en Desarrollo (GED) para poder promover intervenciones claras y bien orientadas hacia la igualdad de género y los derechos de las mujeres (MAEC, 2018a).

Otro de los factores que ha influido en la consolidación del enfoque de género en la Cooperación Española para el Desarrollo han sido los efectos económicos ocasionados por la crisis económica internacional de 2008 , afectando directamente el porcentaje de Ayuda Oficial al Desarrollo destinada 
por la cooperación española, llegando a su cifra más baja de 0,19\% en 2017. Además de la crisis se observa una falta de compromiso político en las cuestiones de promoción de igualdad de género, ya que como hemos podido observar, según el partido político de turno, son las prioridades de los recursos que se destinan a las cuestiones de igualdad de género tanto a nivel nacional como internacional, variando considerablemente de un partido político a otro (Espinoza, 2014, Tomas, 2013). Así lo señala el último Informe de 2018 sobre el Enfoque de Género en la Política de Cooperación Española, al enfatizar lo siguiente:

"A pesar de que la Cooperación Española (CE) ha expresado su compromiso y voluntad de considerar como actuación prioritaria los derechos de las mujeres y la igualdad de género, así como su compromiso con organismos como onu Mujeres, se advierte que el peso de ambas cuestiones ha descendido de modo relevante y específicamente en términos presupuestarios y de AOD. En tal sentido, el presupuesto asignado a la orientación 5: "Promover los derechos de las mujeres y la igualdad de género" - establecida en el IV Plan Director de la Cooperación Española 2013-3016 - ha sido el más exiguo en 2013 y 2014 (21 millones de euros, $1,64 \%$ del total de la AOD)" (MAEC, 2018a).

En este sentido, se considera imprescindible que haya una voluntad y compromiso político, en todos los partidos políticos, para que se mantenga presupuestal e institucionalmente las acciones promovidas por la cooperación española para el desarrollo en materia de igualdad de género.

Según un estudio de Raquel Tomás, existen tres aspectos críticos que podrían condicionar la integración de la perspectiva de género en las políticas de cooperación española al desarrollo, así como el buen funcionamiento de la Agencia Española de Cooperación Internacional para el Desarrollo. siendo estos los siguientes (Tomás, 2013):

1) El mandato político y el compromiso financiero.

2) La cultura institucional.

3) La estructura organizativa y otros aspectos claves de la organización interna.

Para mejorar estos aspectos críticos, la investigadora propone una serie de recomendaciones para mejorar y consolidar la incorporación del enfoque transversal de género en la Cooperación Española al Desarrollo son las siguientes (Tomás, 2013):

1. En relación al mandato político y el compromiso financiero:

- Recomienda velar por una mayor coherencia entre la voluntad política, el marco normativo regulador y la práctica institucional.

- Diseñar y aplicar directrices de género en todo el ciclo de los marcos de asociación, definiendo resultados e indicadores que permitan medir durante la vigencia del acuerdo los progresos del país socio en materia de igualdad. 
- Difundir la guía básica para la transversalización del enfoque de género en la programación operativa y definir su uso como obligatorio por las oficinas y los/as técnicos/as país, donde se va a realizar este ejercicio de programación.

- Proporcionar directrices presupuestarias sobre el trabajo de género, tanto en la sede como en el terreno, formalizando que se reserve el 15\% del presupuesto de todos los instrumentos de la Cooperación Española para la Inclusión del Enfoque de Género, tanto en las convocatorias de ONGD (abierta y permanente, convenios y proyectos), como en los presupuestos bilaterales y los fondos o programas de organismos multilaterales.

- Dotar anualmente al Departamento Sectorial y de Género, al igual que al resto de los departamentos geográficos, de un presupuesto propio para asegurar la transversalidad en todos los sectores e instrumentos con los que se trabaja en la organización.

2.En relación a la Cultura Institucional:

- Crear grupos de trabajo de género horizontales, formales y no-formales, entre los ámbitos sectoriales, geográficos y programáticos para avanzar hacia una cultura más flexible y matricial.

- Elaborar un diagnóstico y posteriormente una estrategia participativa de cambio institucional, que se base en una herramienta de gestión del conocimiento como estrategia para generar el cambio.

- Elaborar una política de incentivos para aquellas personas que logren avances en materia de igualdad de género, según los objetivos establecidos en los presupuestos y en el Plan de Acción de Género de la AECID.

- Fomentar y apoyar la implementación de las políticas de conciliación, desarrolladas por el Departamento de Recursos Humanos, Conciliación y Servicios Generales de la AECID.

- Facilitar la participación de todo el personal en las actividades de sensibilización de los días 8 de marzo y 25 de noviembre, limitando las reuniones de trabajo y motivando a los equipos a participar.

3. En relación a la Estructura Organizativa y otros aspectos de organización interna:

- Elaborar un diagnóstico de necesidades formativas en la sede y en el terreno y diseñar un plan de formación en género y desarrollo, adaptado a las necesidades de la sede y el terreno, y vinculado a los compromisos internacionales y nacionales en materia de igualdad y de eficacia de la ayuda.

- Realizar al menos dos actividades de sensibilización con todo el personal de la AECID, en el marco del 8 de marzo y el 25 de noviembre.

- Crear plazas regularizadas de expertas/os de género en la sede y en las 
unidades de género de las отс para fortalecer la estructura de la sede y el terreno con personal especializado, aparte de contar con la asesoría de expertas en temas específicos cuando sea necesario.

Otras recomendaciones hechas por Manuel Iglesia-Caruncho nos sugieren (Iglesia-Caruncho, 2007):

- Aumentar el presupuestario hasta llegar al 0,7\% de la RNB y garantizar su buen uso para que la inversión sea eficaz.

- Acompasar los incrementos cuantitativos con el aumento de la capacidad de absorción del sistema de cooperación español.

- Fortalecer el cambio institucional con la reforma de la Agencia Española de Cooperación Internacional y a un mayor alineamiento con la Declaración de París.

- Olvidar la necesidad de la especialización geográfica y sectorial, concentrando los recursos donde más puedan aportar.

- Reforzar la comunicación y la sensibilización ciudadana para que la sociedad mantenga su apoyo a la política de desarrollo y cooperación.

- Por su parte, el Grupo de Trabajo de Género y Desarrollo, del Consejo de Cooperación al Desarrollo, en su informe sobre el enfoque de género en la Política de Cooperación Española recomienda lo siguiente (MAEC, 2018a):

i. Mantener la estrategia dual dirigida a la realización de acciones específicas y de fomento de procesos de empoderamiento de todas las mujeres y niñas, así como a la transversalización de la igualdad de género en todos los proyectos, áreas y programas, teniendo siempre en cuenta que las acciones deben dirigirse a toda la sociedad y no solo específicamente a las mujeres.

ii. Mejorar los mecanismos de información y control de manera que se garanticen datos apropiados, para efectuar los diagnósticos adecuados, así como para el seguimiento sistemático y financiar evaluaciones rigurosas que ayuden a entender lo que funciona y lo que no. Estas medidas, así como el contar con datos estadísticos desglosados por sexo y mecanismos de información y control que permitan evitar posibles errores en términos de clasificación de intervenciones son aspectos fundamentales si se quieren ver progresos reales en este ámbito.

iii. Mantener una cooperación al desarrollo que combine la apuesta por la construcción de una estructura institucional adecuada, con el apoyo sostenido de todos los socios, principalmente organizaciones de mujeres y organizaciones feministas y su labor clave para el mantenimiento de estos avances e impulsar nuevos logros. En este sentido, la especialización del personal, la elaboración de instrumentos, protocolos, guías y redes son elementos indispensables. Se considera que puede ser un buen momento para 
actualizar la Estrategia de "Género en Desarrollo" de la Cooperación Española.

iv. Dotar de presupuesto adecuado y suficiente al sector. Se propone, por tanto, ir recuperando de forma progresiva los compromisos y presupuestos destinados a la igualdad y los derechos de las mujeres (género en desarrollo y salud sexual y reproductiva).

v. Incrementar la coordinación entre los documentos estratégicos de igualdad (Plan Estratégico de Igualdad de Oportunidades) y de cooperación (Plan Director), así como su alineamiento con la Agenda 2030 de Desarrollo Sostenible. Así se puede incrementar la coherencia y eficacia de ambas políticas.

vi. Consolidar las redes sectoriales, especialmente la red de género de la AECID y su potencial papel de motor de mesas de género en terreno. En este sentido, se observa la necesidad de mejorar las capacidades técnicas, la formación y la especialización de todo el personal, tanto en sede como en el terreno. vii. Reforzar la presencia activa en espacios de coordinación desde sede y desde terreno.

viii. Continuar con el desarrollo de las redes sectoriales y avanzar en la comunicación sede-terreno y en la gestión del conocimiento, aprovechando la plataforma y las redes de conocimiento como herramienta interna de comunicación para realizar buenos análisis de género. ix. Realizar, por parte de Dirección General de Políticas de Desarrollo Sostenible (DGPOLDES), un análisis sistemático de los resultados de las políticas de género de los últimos años, así como de los factores que puedan estar determinando el éxito y la eficacia de la CE para promover la igualdad de las mujeres y las niñas.

Como podemos observar, todas estas propuestas coinciden en muchos de los aspectos a mejorar, entre los que podemos destacar la formación y sensibilización del personal que trabaja en la puesta en marcha de los planes y acciones, establecidos en los planes de la Cooperación Española al Desarrollo. Su formación y sensibilización con las cuestiones de género son claves para lograr los objetivos establecidos en esta materia, así como su compromiso con la igualdad de género y con la misión de los organismos y dependencias en las que laboran. El incremento presupuestal a los organismos y dependencias de la Cooperación Española al Desarrollo para el logro de los objetivos, con independencia del partido político de turno, es otro de los factores claves para fomentar las estrategias de igualdad tanto a nivel nacional como internacional, así como el incremento del porcentaje de Ayuda Oficial al Desarrollo, en consonancia con los compromisos internacionales adquiridos. La coordinación entre las distintas instancias y dependencias que conforman la Cooperación Española al Desarrollo con los documentos 
establecidos para fomentar la igualdad, son fundamentales para lograr los objetivos plasmados en los Planes y Directrices de la Cooperación para el Desarrollo Española. Mejorar las estrategias de implementación del enfoque transversal de género que sean efectivos y eficaces en las políticas públicas de los países socios. Y sobre todo que haya voluntad política para elevar la igualdad de género y los derechos de las mujeres al nivel de prioridad nacional y de referencia internacional, en consonancia con la legislación nacional y los compromisos internacionales, entre ellos los contenidos en la Agenda 2030 para el Desarrollo Sostenible.

\section{Conclusiones}

La incorporación del enfoque de género en todos los planes y políticas tanto sociales como económicas y medioambientales, es clave para la generación de desarrollo. Así lo ha puesto de manifesto en la Agenda 2030 para el Desarrollo Sostenible, al enfatizar que sin la participación activa de la mujer es imposible generar el desarrollo sostenible que tanto se requiere.

Por su parte, la Agencia Española de Cooperación Internacional para el Desarrollo reconoce la importancia que tiene la incorporación del enfoque de género en las estrategias de cooperación internacional para el desarrollo, al manifestar que la igualdad entre mujeres y hombres es la base del desarrollo; que es necesario reconocer que existe una situación de desigualdad social entre mujeres y hombres con respecto a las actividades que desempeñan, a su acceso a recursos y el control de los mismos y a su participación en la toma de decisiones; que las estrategias de desarrollo no son neutras en relación con el género, al tener consecuencias positivas y negativas diferenciadas para hombres y mujeres; que las acciones de desarrollo son más eficaces si tienen en cuenta las diferencias y desigualdades entre mujeres y hombres; que las relaciones de género interactúan simultáneamente con otras características o condiciones que pueden duplicar o triplicar las desigualdades y discriminaciones como el origen étnico o nacional de las personas, el grupo social, la edad o la orientación sexual, entre otras; que pese a lo que se cree, las mujeres no son un grupo vulnerable (representan más de la mitad de la población mundial), pero debido a las múltiples discriminaciones de las que son objeto, están en condiciones de exclusión y a veces mayor vulnerabilidad; que los hombres son también un elemento clave para el cambio en las relaciones de género. Es imprescindible trabajar con ellos para eliminar las causas estructurales de la desigualdad de género y modificar la percepción 
"tradicional" que tanto hombres como mujeres tienen de su "rol" de género.

Por todo ello, la incorporación de las cuestiones de igualdad de género en la cooperación española ha sido prioritaria desde sus comienzos en la década de los '90.

Hemos podido observar cómo legislativamente se ha ido incorporando la igualdad de género para su puesta en marcha tanto a nivel nacional como internacional, a través de la cooperación al desarrollo. La Estrategia de "Género en Desarrollo" de la Cooperación Española, el Plan de Actuación Sectorial de Género y Desarrollo de la AECID (PAS), el Documento Sectorial Perspectivas y Recomendaciones del Sector Género en Desarrollo de la AEcid, el Plan de Acción Mujeres y Construcción de la Paz de la Cooperación Española, los Planes Directores de la Cooperación Española y la Guía de la AECiD para la Transversalización de Género respaldados por Ley 3/2007 para la Igualdad Efectiva de Mujeres y Hombres (LоIенM), han posicionado la igualdad de género y el empoderamiento de las mujeres como una prioridad nacional.

Hemos analizado también cómo la igualdad de género se ha implementado en la Cooperación Española al Desarrollo bajo diferentes enfoques, siendo el enfoque Mujeres en el Desarrollo (MED) y enfoque Género en el Desarrollo (GED), bajo estrategias de transversalización de este último enfoque, los más utilizados. Sin embargo, hemos podido observar también cómo la estrategia de transversalización del enfoque de género no ha dado los resultados esperados, al no generar un impacto real en las políticas de igualdad de los países socios. Por ello, una de las recomendaciones clave para mejorar las acciones de la cooperación al desarrollo con enfoque de género es mantener una clara distinción entre los enfoques utilizados para que las intervenciones sean claras y bien orientadas, mejorando los resultados en los programas implementados y, por ende, la mejora de la calidad de la vida de las mujeres beneficiadas por dichos programas de desarrollo.

Sin duda, la participación activa de España en los foros y eventos internacionales para la promoción de la igualdad de género han contribuido a la mejora de las políticas nacionales para estar en consonancia con los compromisos internacionales. Prueba de ello es el liderazgo de España en la Agenda de Mujeres, Paz y Seguridad como una herramienta clave para apoyar la incorporación del enfoque transversal de género en las políticas públicas, involucrando a todos los sectores de la población. Así como los rankings internacionales donde sitúan a España por encima de la media tanto europea como internacional en las cuestiones de género: 9o a nivel europeo según el Índice Europeo de la Igualdad de Género del 2019; 80 a nivel mundial el Global Gender Gap Report 2020, y el 23o de 129 países que son evaluados en el Índice de Género de los Objetivos 
del Desarrollo Sostenible (SDG Gender Index/ Equal Measures 2030).

Estas cuestiones, así como los resultados obtenidos en los numerosos programas internacionales desarrollados bajo el enfoque de género, son una muestra de la importancia que tienen las cuestiones de género para el gobierno español.Sin embargo, tras analizar en detalle los aspectos claves tanto institucionales como operacionales de las cuestiones relativas a la igualdad de género en la Cooperación Española al Desarrollo, vemos numerosas y diversas áreas de mejora. Entre las que hemos podemos destacar: la falta de formación, liderazgo y compromiso del personal técnico de la Agencia Española de Cooperación Internacional para el Desarrollo, que asumen la responsabilidad política de promoción de la equidad de género en los programas y proyectos; la ausencia de la incorporación real del enfoque transversal de género en la formulación, ejecución, seguimiento y evaluación de las intervenciones realizadas que genere cambios reales en la vida de las mujeres beneficiarias de los programas de cooperación; la financiación de las instituciones y dependencias del sistema de cooperación al desarrollo español, así como la cooperación de dichas dependencias para el logro de los objetivos establecidos en los planes y directrices de la cooperación española; la variación del porcentaje de la ayuda oficial al desarrollo, en función de las prioridades del gobierno de turno de los recortes nacionales por cuestiones externas, como la crisis de 2008; la falta de voluntad política para dotar presupuestaria e institucionalmente a las acciones de igualdad de género dentro de la Cooperación Española para el Desarrollo, entre otras.

Para ello, hemos podido analizar varias propuestas hechas por investigadores y expertos en la igualdad de género en las estrategias de desarrollo, los cuales coinciden en avanzar hacia la formación y sensibilización del personal que trabaja en la puesta en marcha de los planes y acciones establecidos en los planes de la Cooperación Española al Desarrollo; hacia un compromiso real con la igualdad de género y con la misión de los organismos y dependencias en las que laboran; hacia el incremento presupuestal a los organismos y dependencias de la Cooperación Española al Desarrollo para el logro de los objetivos, con independencia del partido político de turno, así como el incremento del porcentaje de Ayuda Oficial al Desarrollo, en consonancia con los compromisos internacionales adquiridos; hacia la coordinación entre las distintas instancias y dependencias que conforman la Cooperación Española al Desarrollo con los documentos establecidos para fomentar la igualdad para lograr los objetivos plasmados en los Planes y Directrices de la Cooperación para el Desarrollo Española; hacia la mejora de las estrategias de implementación del enfoque transversal de género que sean efectivos y eficaces en las políticas públicas de los países socios, y sobre 
todo trabajar para que haya voluntad política para poder elevar la igualdad de género y los derechos de las mujeres al nivel de prioridad nacional y de referencia internacional, en consonancia con los compromisos internacionales, y concretamente en lo contenido en la Agenda 2030 para el Desarrollo Sostenible.
Con todo ello, es evidente que el trabajo que tiene la Cooperación Española para el Desarrollo en este sentido es muy grande, pero cuenta con una base legislativa sólida que puede soportar los cambios requeridos y una sociedad comprometida con las cuestiones relativas a la igualdad de género, tanto dentro como fuera del territorio español.

\section{Bibliografía}

ACNUDH (2006). "Preguntas frecuentes sobre el Enfoque de los Derechos Humanos en la Cooperación para el Desarrollo". Oficina del Alto Comisionado de las Naciones Unidas para los Derechos Humanos. Palais des Nations, 8-14 avenue de la Paix, CH-1211 Ginebra 10, Suiza. Ps. 50.

AECID (2015). "Guía de la AECID para la Transversalización del Enfoque de Género". Madrid, España. pp. 146.

Alonso,J.A. (Dir.) (2015). “Movilizando los recursos y los medios de apoyo para hacer realidad la Agenda de Desarrollo post-2015". Secretaría General de Cooperación Internacional para el Desarrollo. Madrid, España. Pp. 113.

Angulo, G. (2015). “España frente a los retos de la Agenda de Desarrollo Sostenible". OXFAM Intermón, UNICEF Comité Español pp. 49.

Arguilés, J.M. (2014). "25 años de evaluación de la política española de cooperación internacional para el desarrollo". Revista de Evaluación de Programas y Políticas Públicas, UNED, Facultad de
Ciencias Económicas y Empresariales, Núm.3. p. 25.

BOE (1998). "Ley de Cooperación Internacional para el Desarrollo”, Núm. 162. Boni, A. (2010). "El sistema español de cooperación internacional al desarrollo”, en La Cooperación Internacional para el Desarrollo, Capitulo III. Cuadernos de Cooperación para el Desarrollo Núm. 1. Centro de Cooperación al Desarrollo, Editorial Universitat Politècnica de València.p. 9.

Boni, A. (1998a) "Real Decreto 23/1998, "Ley de Cooperación Internacional para el Desarrollo”, pág. 22756, Núm. 162.

Espinosa, J. (2014). "La igualdad de género en la cooperación española y en la iniciativa Marca España. Abordajes e inconsistencias en tiempos de crisis”. Investigaciones feministas, ISSN 2171-6080, №. 5, (Ejemplar dedicado a: Monográfico: Políticas públicas en tiempos de crisis. Un análisis desde la perspectiva de género), pp. 259-288.

Espinosa, J. (2013). "La evaluación sensible al género: una herramienta para 
mejorar la calidad de la ayuda" en Leyra Fatou Begoña y Pérez Camporeale Ana M. (eds.), Integración del enfoque de género en políticas, planes y proyectos para el desarrollo: avances, retrocesos, desafíos y propuestas para una adecuada implementación. Universidad Complutense. Instituto Complutense de Estudios Internacionales, ISBN: 978-84-695-7310-5.

Espinosa, J. (2007). "La perspectiva de género en la evaluación de programas de desarrollo internacional" Evaluación en la cooperación para el desarrollo. Cuaderno 33 de Escuela Diplomática.

Fundación Mujeres (2003). "Guía para la elaboración de proyectos desde una perspectiva de género". Fondo Social Europeo y Gobierno del Principado de Asturias.

Gobierno de España (2018), "Informe de España para el Examen Nacional Voluntario 2018”. Gobierno de España, Madrid, pp. 193.

Gómez, M. (2010). “El Sistema Español de Cooperación Internacional al Desarrollo,"en La Cooperación Internacional para el Desarrollo, Capitulo III.Cuadernos de Cooperación para el Desarrollo Núm.

1. Centro de Cooperación al Desarrollo, Editorial Universitat Politècnica de València. pp.77-89.

Iglesia-Caruncho, M. (coord.) (2007). "Avances y Retos de la Cooperación Española Reflexiones para una Década”. Fundación Carolina, Madrid. P. 640.

Jiménez, L. (2015).“Variables influyentes en la transversalización de la perspectiva de género de proyectos financiados por la Agencia Española de Cooperación
Internacional para el Desarrollo", Revista de Evaluación de Programas y Políticas Públicas, ISSN-e 2340-8464, №. 5, págs. 1-34.

Jiménez, L. (2014). "El uso transversal de la perspectiva de género en proyectos financiados por la Agencia Española de Cooperación Internacional para el Desarrollo con aportaciones de técnicos-as y protagonistas locales (en Ecuador, Mozambique y Senegal)" Tesis doctoral dirigida por Eusebio Medina García (dir. tes.). Universidad de Extremadura (2014).

Leyra, B. y Pérez, A.M. (eds.) (2013). "Integración del enfoque de género en políticas, planes y proyectos para el desarrollo: avances, retrocesos, desafíos y propuestas para una adecuada implementación. Universidad Complutense. Instituto Complutense de Estudios Internacionales, ISBN: 978-84-695-7310-5.

MAEC (2020) "Igualdad en la política exterior y de cooperación para el desarrollo”, Ministerio de Asuntos Exteriores, Unión Europea y Cooperación, Gobierno de España, p. 12.

MAEC (2018). "La nueva métrica de la cooperación para el Desarrollo: el apoyo oficial total para el desarrollo sostenible (AOTDS/TOSSD) y su aplicación a España”, Secretaría de Estado de Cooperación Internacional y para Iberoamérica y el Caribe, Dirección General de Políticas de Desarrollo Sostenible, Madrid, España.pp. 122.

MAEC (2018a). "Informe sobre el enfoque de género en la política de Cooperación Española”, Consejo de Cooperación al Desarrollo. Grupo de Trabajo 
de Género y Desarrollo. pp. 5.MAEC (2007), "Estrategia de Género en Desarrollo de la Cooperación Española”, Secretaría de Estado de Cooperación Internacional, Dirección General de Planificación y Evaluación de Políticas para el Desarrollo, Madrid, España. pp. 158.

MAEC (2015)."Compromiso Universal por un Desarrollo Humano y Sostenible: posición española para la agenda post 2015”, Secretaría General de Cooperación Internacional para el Desarrollo, Madrid, España. pp. 80.

MAEC (2013). "Plan Director de la Cooperación Española 2013/2016”, Secretaría General de Cooperación Internacional para el Desarrollo, Madrid, España. Pp. 151.

Molina, Ma..E. (2015). "Los estudios de género y desarrollo (EGED) en construcción: desafíos y oportunidades para la cooperación universitaria al desarrollo (CUD) en España”. Aportaciones a la investigación sobre mujeres y género: V Congreso Universitario Internacional "Investigación y Género: Sevilla, 3 y 4 de julio de 2014 / coord. por Rosa Casado Mejía, Consuelo Flecha García, Ana Guil Bozal, M. Teresa Padilla-Carmona, Isabel Vázquez Bermúdez, María del Rocío Martínez Torres, ISBN 9788494312038, pp. 956-977.

Molina, Mạ.E. (2013). "Políticas de Cooperación Universitaria al Desarrollo con Perspectiva De Género. La Red GEDEA (Género en Desarrollo y Eficacia de la Ayuda) como buena práctica de la CUD”, en Leyra Fatou, B. y Pérez Camporeale, A.M. (eds.), Integración del enfoque de género en políticas, planes y proyectos para el desarrollo: avances, retrocesos, desafíos y propuestas para una adecuada implementación. Universidad Complutense. Instituto Complutense de Estudios Internacionales, ISBN: 978-84-695-7310-5.

OXFAM Intermón y UNICEF Comité Español (2015). "España frente a los retos de la Agenda de Desarrollo Sostenible”. Madrid, España, pp. 49.

Pedrajas, M. (2014). “Las prioridades de la posición española para la Agenda de desarrollo post2015", Información Comercial Española, ICE: Revista de economía, ISSN 0019-977X, № 880, (Ejemplar dedicado a: Globalización y Desarrollo), pp. 127-140.

Sotillo, J.A. (2006). Un lugar en el mundola política de desarrollo de la Unión Europea. Instituto Universitario de Desarrollo y Cooperación (IUDC). Madrid: Catarata.

Tomás, R. (2013). "El enfoque de género en la cooperación española: avances y cambios frente a viejos desafíos institucionales”, en Leyra Fatou, B. y Pérez Camporeale, A.M. (eds.), Integración del enfoque de género en politicas, planes y proyectos para el desarrollo: avances, retrocesos, desafíos y propuestas para una adecuada implementación. Universidad Complutense. Instituto Complutense de Estudios Internacionales, ISBN: 978-84-695-7310-5.

VENRO (2009). "Género en la Cooperación para el Desarrollo”. Marco de Orientación. p. 31. 